\title{
What is the most sensitive non-invasive strategy for the diagnosis of intracranial aneurysms?
}

In the paper by White et al (this issue, pp 322-328) ${ }^{1}$ noninvasive tests for the detection of intracranial aneurysms are evaluated. Combining these tests is shown to improve the diagnostic performance, the combination of power Doppler and CT angiography having the greatest sensitivity $(0.83)$ for aneurysm detection with a strong agreement with the gold standard of digital subtraction angiography (к 0.84 ).

This paper shows that the performance of these non-invasive imaging techniques is improving. One of the concerns raised is that small aneurysms, of $5 \mathrm{~mm}$ or less, cannot be reliably identified. This concern has been diluted by findings of the International Study on Unruptured Intracranial Aneurysms (ISUIA) ${ }^{2}$ which indicated that aneurysms under the size of $10 \mathrm{~mm}$ have a very low rate of spontaneous rupture (about $0.05 \%$ /year) and do not justify the risks of treatment in most cases. ${ }^{3}$ While the technology progresses, what remains to be established are the indications for their application, particularly in the context of the very low complication rate $(0.07 \%$ risk of permanent neurological deficit) for conventional digital subtraction angiography (DSA), ${ }^{4}$ which does require a short inpatient stay.

What are the indications for imaging for suspected intracranial aneurysms? These can be divided into two groups - those patients who have had a subarachnoid haemorrhage $(\mathrm{SAH})$ and those who have not. For patients with an SAH, the indications are firstly to investigate the cause of an acute bleed, secondly to follow the progress of other unruptured aneurysms in the patient, and thirdly, surveillance for those who have received endovascular treatment. Currently, investigation of acute SAH requires conventional DSA. The consequences of inadequate information in the investigation of a ruptured aneurysm is considerable, and a 0.83 sensitivity for non-invasive imaging is not sufficient. The surgical technique is also directed by the anatomy of the aneurysm as shown on DSA. The decision to treat unruptured aneurysms detected at the time of DSA after SAH can usually be taken at the time after recovery from treatment of the ruptured aneurysm. There is rarely an indication to follow such aneurysms. The risk of rupture is $0.5 \%$ /aneurysm/year and the decision to treat therefore depends on the age of the patient and the risk of the treatment, taking into account the size and location of the aneurysm. In general, small aneurysms in elderly people do not require treatment (and there is no indication for repeat imaging) whereas younger patients with aneurysms above $10 \mathrm{~mm}$ should be counselled and offered treatment. In terms of endovascular surveillance DSA is the standard. Non-invasive imaging that can detect a residual aneurysm neck and refilling would realise considerable cost savings.

For patients without $\mathrm{SAH}$, recent evidence has indicated that there is no benefit in screening asymptomatic relatives. ${ }^{5}$ The criteria for a satisfactory screening test are not fulfilled, with potential morbidity of treatment exceeding any potential benefit. Asymptomatic lesions found incidentally on imaging which approach $10 \mathrm{~mm}$ in size may lend themselves to non-invasive surveillance.

Finally, does non-invasive imaging offer an advantage over conventional DSA in addition to the negligible complication rate? In certain circumstances, especially giant aneurysms, CT angiography can offer benefits in defining the relation between the aneurysm, respective cerebral arteries, and skull base, assisting in planning and risk assessment.

In summary, the paper by White $e t$ al ${ }^{1}$ illustrates the considerable progress that has been made in refining non-invasive imaging of cerebral aneurysms. In addition to refining the sensitivity of these techniques, future developments need to parallel modern ideas on clinical indications for aneurysm detection and surveillance.

PJ HUTCHINSON PJ KIRKPATRICK

Department of Neurosurgery, University of Cambridge, Box 167, Addenbrooke's Hospital, Hills Road, Cambridge CB2 2QQ, UK

Correspondence to: Mr P J Hutchinson, p.hutch@which.net

1 White PM, Teasdale, E, Wardlaw JM, et al. What is the most sensitive noninvasive imaging strategy for the diagnosis of intracranial aneurysms? $\mathcal{F}$ invasive imaging strategy for the diagnosis
Neurol Neurosurg Psychiatry 2001;71:322-8.

2 Unruptured intracranial aneurysms-risk of rupture and risks of surgical intervention. International Study of Unruptured Intracranial Aneurysms. N Engl f Med 1998;339:1725-33.

3 Kirkpatrick PJ. Time to reconsider treatment options for intracranial aneurysms. Lancet 1999;353:942-43.

4 Cloft HJ, Joseph GJ, Dion JE. Risk of cerebral angiography in patients with subarachnoid haemorrhage, cerebral aneurysm, and arteriovenous malformation: a meta-analysis. Stroke 1999;30:317-20.

5 Kirkpatrick PJ, McConnell RS. Screening for familial intracranial aneurysms. BMF 1999;319:1512-3.

\section{Divining dementia}

By the time you reach your 65th birthday, you will have over a $5 \%$ chance of having dementia. ${ }^{1}$ Or will you? The prevalence rates of dementia as $5 \%$ of the over 65 s and $20 \%$ of the over 80 s are often quoted, but these raw figures belie a more complex interrelation between the risk of developing dementia and the individual person. This issue of the fournal includes two articles that tackle the problem of predicting dementia. One, by Helmer et al (pp 303-309) ${ }^{2}$ investigates the relation between dementia and premorbid occupation. The second, by Waite et al (pp 296-302), ${ }^{3}$ explores the utility of preclinical syndromes that may presage dementia. These issues pose ethical and social 
problems, but may be important in improving the care of patients with dementia

The suggestion that high premorbid intellect protects against the development of dementia has been elegantly demonstrated in the Nun study, which found that individuals who wrote grammatically complex sentences and demonstrated high idea density (average number of ideas per 10 words) had better cognitive function and lower rates of dementia six decades later. ${ }^{4}$ Whether premorbid intellect is negatively correlated with later cognitive impairment because increased neuronal branching and connectivity related to learning are protective, or because individuals just have further to fall, remains unclear.

Although Helmer et al reported that female farmers seemed to be at greater risk of developing dementia with parkinsonism, occupation was a poor predictor of risk for developing Alzheimer's disease. At first this seems counterintuitive, as cognitive functioning and occupation are closely linked. However, other factors may play a part in later development of dementia - for example, lower ongoing intellectual stimulation (the use it or lose it paradigm) and lifestyle and occupational factors that may be implicated in the aetiology of dementia. Some occupational risk factors such as repeated head injury have been identified although others (Helmer et al suggest pesticide use among farmers) remain speculative.

Preclincial syndromes that indicate high risk of developing dementia may be important to recognise. Use of cholinesterase inhibitors such as donepezil, rivastigmine or galantamine in those with subclinical dementia may lead to better treatment success than is currently the case when treating established disease. Although recently approved by the National Institute for Clinical Excellence in the United Kingdom, cholinesterase inhibitors result in only marginal improvements in established mild to moderate Alzheimer's disease. For example, you would need to treat 17 people with rivastigmine rather than placebo for one additional patient to have a 4 point improvement on the 70 point ADAS cognitive assessment scale after 26 weeks. ${ }^{5}$

Other benefits may also result from early diagnosis: the ability to plan for your illness (for example, writing a will, advance directive, and an enduring power of attorney while capacity exists), taking the trip of a lifetime, talking to estranged relatives and friends, and fulfilling other longstanding ambitions. Early diagnosis may also help to remove the atmosphere of secrecy and stigma the label still confers.

Waite $e t a l^{4}$ identify a problem with the current role of preclinical syndromes in predicting dementia; the relatively low positive predictive value. The current answer to the question "if I have a preclinical syndrome what are the chances of me developing dementia in the next 3 years?" is "around 20\%". This leads to an ethical conundrum; whether or not to screen people for preclinical syndromes, as doing so should mean having to inform them of the result despite the high false positive rate. It is essential to minimise false positives when diagnosing dementia because of the significance of the diagnosis and, as Waite et $a l^{3}$ suggest, screening currently remains a research tool.

The need to identify accurately people with early or preclinical dementia has never been more acute. The most important approaches to achieve this include changing illness behaviour by increasing awareness and reducing the stigma of dementia among the public, promoting early recognition and referral by general practitioners and building "risk profiles" that are predictive of dementia.

J WARNER

Imperial College School of Medicine, Paterson Centre, 20 South Wharf Road, London W2 1PD, UK

j.warner@ic.ac.uk

1 Lobo A, Launer LJ, Fratiglioni L, et al. Prevalence of dementia and major subtypes in Europe: A collaborative study of population-based cohorts. Neurologic Diseases in the Elderly Research Group. Neurology. 54 (11 Suppl 5): $\mathrm{S} 4-9,2000$

2 Helmer C, Letenneur L, Rouch I, et al. Occupation during life and risk of dementia in French elderly community residents. I Neurol Neurosurg Psychiatry 2001;71:303-9.

3 Waite LM, Broe GA, Grayson DA, et al. Preclinical syndromes predict dementia: the Sydney older persons study. F Neurol Neurosurg Psychiatry 2001;71:296-302.

4 Snowdon DA, Kemper SJ, Mortimer JA, et al. Linguistic ability in early life and cognitive function and Alzheimer's disease in late life. Findings from the Nun Study $7 A M A 1996 ; 275: 528-32$,

5 Birks J, Grimley Evans J, Iakovidou V, et al. Rivastigmine for Alzheimer's disease (Cochrane review). Oxford: The Cochrane Library, 2001:1, update software. 